\title{
Prominent Inhibitory Projections Guide Sensorimotor Computation: An Invertebrate Perspective
}

\author{
Samantha Hughes* and Tansu Celikel*
}

From single-cell organisms to complex neural networks, all evolved to provide control solutions to generate context- and goal-specific actions. Neural circuits performing sensorimotor computation to drive navigation employ inhibitory control as a gating mechanism as they hierarchically transform (multi)sensory information into motor actions. Here, the focus is on this literature to critically discuss the proposition that prominent inhibitory projections form sensorimotor circuits. After reviewing the neural circuits of navigation across various invertebrate species, it is argued that with increased neural circuit complexity and the emergence of parallel computations, inhibitory circuits acquire new functions. The contribution of inhibitory neurotransmission for navigation goes beyond shaping the communication that drives motor neurons, and instead includes encoding of emergent sensorimotor representations. A mechanistic understanding of the neural circuits performing sensorimotor computations in invertebrates will unravel the minimum circuit requirements driving adaptive navigation.

\section{Introduction}

Life's aim is an act, not a thought. The brain seems a thoroughfare for nerve-action passing its way to the motor animal (Sir Charles Scott Sherrington, The Brain and Its Mechanisms, 1933)

If generating an action is the primary goal of the nervous system, studying neural computations that lead to motor behaviors across the evolutionary tree will ultimately unravel

Dr. S. Hughes

HAN BioCentre

HAN University of Applied Sciences

Nijmegen 6525EM, The Netherlands

E-mail: samantha.hughes@han.nl

\section{Prof. T. Celikel}

Department of Neurophysiology

Donders Institute for Brain Cognition and Behaviour

Radboud University

Nijmegen 6525A), The Netherlands

E-mail: celikel@neurophysiology.nl

The ORCID identification number(s) for the author(s) of this article can be found under https://doi.org/10.1002/bies.201900088.

(C) 2019 The Authors. BioEssays Published by Wiley Periodicals, Inc. This is an open access article under the terms of the Creative Commons Attribution License, which permits use, distribution and reproduction in any medium, provided the original work is properly cited.

DOI: 10.1002/bies.201900088 the (un)common solutions that seemingly distinct neural network formations provide to perform behaviorally relevant adaptive computations. As navigation itself has evolved within species' habitats and the transformed body schemes, the composition of neural networks has become compliant to their new constraints. This sometimes leads them to acquire novel sensory inputs and often changes how effectors (e.g., muscles) interact with the environment. With increasingly complex circuit formations throughout the evolution, a preserved circuit motif might have allowed increasingly complex circuit formations and adaptive behaviors to evolve.

Navigation is a whole-brain computation. In animals with a central nervous system, it involves both egocentric (i.e., self-centered) and allocentric (i.e., world-centered) encoding of sensory information. The endpoint of this computation is an egocentric action sequence that allows the interaction with the surrounding world. World-centered encoding of sensory information necessarily requires mapping sensory information with respect to navigational goals and contextual information. Thus, self-centered representation must be transformed and integrated with previously acquired sensory and perceptual experiences in the form of cognitive maps. Therefore, complex navigation is a whole-brain computation that consolidates information processing across a sensation-perception-action triangle while engaging memories and recruiting executive control.

Navigation can also be a task for a single cell; mobility can be generated as a response to local sensory information by individual specialized "sensorimotor cells" that contain both sensory receptors and an organ for generating motion. Eukaryotes with cilia and ciliated sponge larvae, for example, employ ciliary motion to navigate their environment in response to a change in illumination, chemical gradients, and gravity. ${ }^{[1,2]}$ It has been speculated that the first neurons evolved to provide fast electrical signal propagation to control navigation, ${ }^{[3]}$ which ultimately gave rise to organisms that employ muscle-based locomotion as cilia evolved into a sensory organ. ${ }^{[4]}$ Independent of whether the mobility is provided by a ciliary organ or a muscle-based effector system, ${ }^{[5,6]}$ coordinating navigation in a context where motor action is generated as a response to current (or recent history of the) sensory information constitutes the basis of sensorimotor integration. ${ }^{[7-9]}$ 
Although sensorimotor computation commonly leads to navigation (as well as other forms of motor control including vocalization and vocal communication ${ }^{[10-14]}$ ), we refrain from discussing the circuits of navigation, which are reviewed elsewhere. ${ }^{[15-20]}$ Here, we focus on the networks that have evolved to provide such sensorimotor control to address general principles of sensorimotor computation from a neural circuit perspective, with the focus being on Caenorhabditis elegans ( $C$. elegans). By focusing our arguments on three key highlights, we show that $C$. elegans, like all animals, require sensorimotor integration to generate action and explore the computational roles of inhibitory neurons that allow adaptive sensorimotor computations. Ultimately, by understanding the mechanistic basis of the neural circuits that perform sensorimotor computations in a simple model organism such as C. elegans, this will help to unravel the minimum circuit requirements that drive adaptive navigation.

\section{All Animals Use Sensorimotor Integration to Generate Context-Specific Actions}

From nematodes to primates, all animals have specialized neural circuits that enable them to integrate the variety of (often multimodal) sensory information to plan an appropriate motor action. Although the complexity of the behavior, dimensionality of the sensory stimulus, and motor repertoire vary greatly, the basic principles of sensorimotor integration are preserved across species.

The simplest form of sensorimotor integration can be observed in taxis, where the motion of the organism is controlled as a direct consequence of a specific sensory stimulus, e.g., temperature for thermotaxis, ${ }^{[21]}$ a light for phototaxis, ${ }^{[22]}$ chemical gradients for chemotaxis, ${ }^{[23]}$ and wind for anemotaxis. ${ }^{[2]}$ As organisms use distinct cues to explore their environments, where and when the sensory stimuli are encountered is crucial in determining animals' motor responses. Although a unimodal sensory stimulus could effectively control taxis, locomotor networks can also be driven by multimodal inputs, thanks in part to the multimodal integration in sensory neurons. Multimodal integration is experiencedependent, at least in mammals. ${ }^{[25]}$ However, experience-dependent adaptive navigation strategies dominate navigation even if unimodal sensory information drives sensorimotor integration. Whether a bat hunts for a mouse using echolocation at night ${ }^{[26,27]}$ or a mouse contextually controls the position of its whiskers in space while foraging its environment in darkness, ${ }^{[7,28]}$ neural circuits reconstruct, and otherwise memorize, the recent history of the sensory input. This time derivative of the incoming sensory input, often in relation to the motor state of the animal, is an important control signal that shapes the motor output of the organism. ${ }^{[29]}$

A major difference between the simple and complex nervous systems is that the former perform navigation without a map. Thus, their motor control strategy is tightly coupled to the sensory information available in their immediate environment, rather than being based on planned (often goal-directed) actions, which require motor planning in the context of landmarks and world-centric coordinates. ${ }^{[30-33]}$ Accordingly, as C. elegans moves through a chemical gradient, it navigates its environment using a biased random walk, ${ }^{[34,35]}$ just like bacteria navigate a chemical gradient, ${ }^{[36]}$ using multiprotein receptor complexes for temporally integrating information. ${ }^{[37]}$ Integrated sensory information can change animals' navigation strategies. ${ }^{[38-40]}$ In C. elegans, the behavior of the nematode in the absence of food will change the navigational state from "searching" to "dispersal," each of which requires a distinct set of sensory neurons. ${ }^{[1,42]}$ The rate of turns is regulated by the sensory experience across time scales as the worm adapts to its environment, e.g., a high number of turns are observed following the initial disappearance of food and if food sparsity continues, the number of turns will eventually decrease to a base level. ${ }^{[41]}$ Consequently, even animals with simple nervous systems are capable of a rapid response to the ongoing changes in the environment, and over time, they adapt to the new sensory state of their surroundings. ${ }^{[41]}$

Consequently, the integration of sensorimotor information generates highly context-specific actions, ${ }^{[43]}$ whether in terms of a quick escape from a predator or navigation in response to food availability. Planned, goal-directed navigation provides a distinct advantage over navigation based on local cues as it allows integration of sensory information across larger spatial domains while introducing (rapid) error correction for the execution of sensorimotor behaviors. With increased mnemonic load, complex neural circuits progressively integrate longterm information storage capability to the circuits that perform sensorimotor integration that constitute the neural basis of perceptual and motor learning. ${ }^{[41,44-49]}$ Neural circuits have thus evolved to execute sensorimotor integration with everincreasing involvement from mnemonic contributions and executive control, for example in the mammalian brain, the circuits that plan, control, execute, and monitor action generation. ${ }^{[50-53]}$

\section{Rudimentary Neural Circuits That Mediate Sensorimotor Computation Employ Hierarchical Information Processing}

One of the basic forms of sensorimotor computation is the activation of the body wall, or skeletal muscles as sensory input from the environment is used to create a reactive control scheme for navigation. In simplified neural circuits, this control scheme involves hierarchical processing of (multi)sensory information by interneurons as action sequences are translated into motor commands by motor neurons, e.g., the fast escape response in fish, ${ }^{[54]}$ the powerful escape action in flies, ${ }^{[55]}$ the reactive escape in lamprey, ${ }^{[56]}$ and the threat response in C. elegans. ${ }^{[57]}$

Motor activity results from the interplay between excitatory and inhibitory interneurons that synapse onto motor neurons in both vertebrates and invertebrates. The small and compact nervous system of $C$. elegans means that only two layers separate sensory neurons from motor neurons (Figure 1a). Here, the premotor interneurons (AVB and PVC) synapse onto excitatory B-type motor neurons to drive forward movement, while alternative interneurons (AVA, AVD, and AVE) connect to the excitatory Atype motor neurons to stimulate reverse movement. ${ }^{[41,44]}$ The role of the inhibitory ( $\gamma$-aminobutyric acid [GABA]) D-type motor neurons is to innervate muscles of the opposing side, enabling 

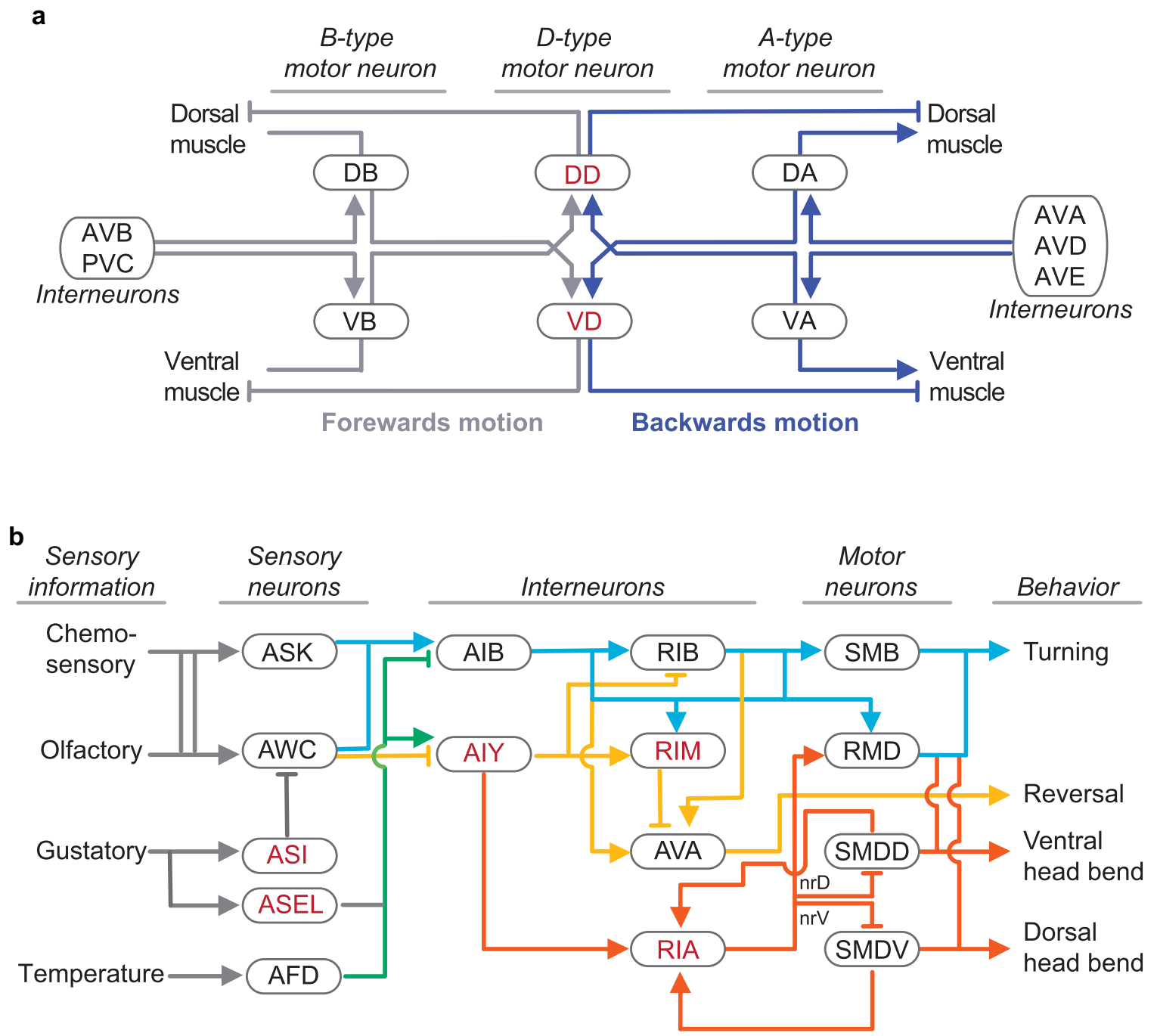

Figure 1. Hierarchical feed-forward information processing for sensorimotor navigation in C. elegans. a) The undulatory forward/backward movement of the animal is driven by contraction and relaxation of muscles on the dorsal (D) and ventral (V) side of the worm. Cholinergic motor neurons (A- and B-type) are postsynaptic to interneurons and drive locomotion while the GABAergic neurons (D-type) inhibit muscle contraction. This is repeated along the length of the animal in a contralateral inhibitory mechanism, i.e., DB motor neurons excite a dorsal muscle at the same time as VD motor neurons inhibit the opposing ventral muscle. ${ }^{[4]}$ Blue lines demonstrate forward movement and gray lines show backward movement, with cell ID labels in red denote inhibitory interneurons. b) The amphid sensory neurons, AWC, ASK, and ASI, direct their synaptic output to the primary layer of interneurons (AIB and AIY) and then to the secondary layer interneurons (RIM, RIB, and RIA). The AVA is a command interneuron and is able to act in parallel to, as well as in concert with, the interneurons. The AIY neuron has a key role in head bending in response to various taxis behaviors via the RIA. The RIA neuron makes reciprocal connections with SMDV and SMDD motor neurons, where the connections are spatially segregated so that the dorsal $\mathrm{nrD}$ region of the RIA innervates SMDD and results in ventral head bends via local calcium ion levels and vice versa for $\mathrm{nrV}$ innervation of SMDV. ${ }^{[41,58-61]}$ Cell ID labels in red denote inhibitory interneurons. Blue lines demonstrate neuronal circuitry that promotes movement, green lines show both excitatory and inhibitory synapses from a sensory stimulus, and yellow lines show inhibitory circuitry. Orange lines indicate the circuitry associated with corollary discharge.

modulation of the contralateral inhibition in the nematode worm. ${ }^{[44]}$ The role of inhibitory neurons is not limited to gating and coordinating motion. Multimodal sensory input is collected and transmitted to the AIY interneuron, where the inhibitory signals and excitatory signals are integrated ${ }^{[41,58]}$ (Figure 1b). The motor output is head turning and navigation across a variety of contexts, including chemotaxis, thermotaxis, and swimming. ${ }^{[41,62-64]}$ Such feed-forward loops allow head movement to be mediated by a single inhibitory neuron, the RIA.
Generation of adaptive, yet robust, behavioral output with limited circuit components would benefit from experience-dependent plasticity of neuronal communication and compartmentalization of computation. ${ }^{[39,65]}$ Indeed, C. elegans displays many aspects of plasticity, including learning and memory, ${ }^{[6-68]}$ in the absence of which navigational trajectories become more random, less ordered, and less directional. ${ }^{[69]}$ For example, during isothermal tracking, where worms migrate toward their cultivation temperature, ${ }^{[70]}$ expression of the calcium sensor ncs-1 in the interneuron AIY 
has a crucial function in influencing experience-dependent learning. Studies in mice have shown that NCS-1 may facilitate learning and memory together with motivational and reward circuits, where overexpression of ncs-1 promotes exploration and acquisition of spatial memory while ncs-1 knockout impairs exploration and long-term memory. ${ }^{[71]}$ As NCS-1 regulates synaptic communication of the presynaptic inhibitory AIY neuron projections in C. elegans, ${ }^{[70,72]}$ targeted regulation of inhibitory neuron communication might enable the emergence of adaptive sensorimotor transformation.

Strikingly, the AIY interneuron has the ability to regulate two different outputs via multiple downstream circuits. ${ }^{[73}$ The inhibitory connections between the AIY interneuron and AIZ provide a means to control the direction of movement, while the excitatory connection between AIY and AIB allows the speed of movement to be modified. ${ }^{[73]}$ In addition, depending on the intensity of a stimulus, AIB is able to modulate behavioral output accordingly. ${ }^{[74]}$

In postsynaptic neurons, compartmentalization is an important computational feature and is likely to be a conserved mechanism for signal processing. ${ }^{[75,76]}$ In $C$. elegans, the axon of the RIA interneurons has several distinct domains (the loop, $\mathrm{nrD}$ and $\mathrm{nrV}$ ) in which dynamic calcium levels result in head movement; SMDV innervates the $n r V$ and displays ventral head bends while SMDD synapses onto nrD, enabling dorsal head bending ${ }^{[75]}$ (Figure 1b). These calcium dynamics were also observed in the presence of levamisole, a nicotinic acetylcholine receptor agonist, suggesting that RIA axonal activity is independent of movement, ${ }^{[75]}$ further arguing that inhibitory RIA neurons represent proprioceptive feedback and/or corollary discharge, also known as efference copy. ${ }^{[59,75]}$

Synapses are dynamically controlled to modulate behavior, and compartmentalization is a level of organization by which different pathways can be activated, suppressed, or otherwise modulated for the generation of behavior. By spatially restricting signaling, as in the $C$. elegans RIA neuron, different subcircuits can be selected from a larger pool of possible circuits, enabling the fine-tuning of a response. ${ }^{[59,68,77,78]}$ Compartmentalized activity has been shown in complex networks including the optic lobe of the fruit fly ${ }^{[79,80]}$ and the starfish. ${ }^{[81,82]}$ Compartmental computations are also preserved in more complex circuits, including the mammalian brain. ${ }^{[83]}$ Thus, mapping the origins (e.g., presynaptic soma location, neuron type, and synapse class) of synapses made onto the postsynaptic neuron will complement these functional studies while enabling a circuit-level description of spatiotemporal integration for sensorimotor transformation.

By spatiotemporally confining neuronal activity to a specific subcellular region, the computational power of neurons can be expanded dramatically. Furthermore, this form of circuit organization allows convergence of synaptic inputs originating from nonoverlapping presynaptic pools of neurons while preserving stimulus selectivity before the neuronal representations can be passed along the neuronal circuits. By forming routine operations of the nervous system, e.g., gain modulation, stimulus selectivity, around feed-forward computations, neuronal representations can be maintained across a broad range of stimuli.

\section{Independent of the Evolutionary Age of the Organism, Inhibitory Neurons Powerfully Shape the Motor Control during Sensorimotor Computation}

It has been known since 1863 that inhibitory neurons regulate motor control, ${ }^{[84]}$ although it was not for another 50 years that the significance of inhibition truly took hold. ${ }^{[85,86]}$ Now, it is clear that inhibition is an important building block of sensorimotor circuits, and is required to establish the correct patterns of motor activity and shape its plasticity ${ }^{[87]}$ and inhibitory circuits are present throughout sensorimotor circuits.

Inhibition circuitry is an important feature of sensorimotor circuits that are required to establish the correct patterns of motor activity to enable locomotion. Sensorimotor inhibition is required to regulate the processing of sensory information via presynaptic pathways and via postsynaptic inputs to neurons that are involved in receptive sensory transmission and control the excitability of motor neurons by directly or indirectly reducing excitation. ${ }^{[88]}$ Stopping or lowering the sensory feedback before it reaches a spinal target is an efficient way to control afferent inputs in different motor tasks. Purkinje cells (PCs) are the sole output from the cerebellar cortex and it is generally accepted that the PCs are important in the fine control of movements. The ability of PCs to contribute to motor control is owing to the fact that, firstly, they are inhibitory neurons and secondly they have a relatively high spontaneous firing frequency that can be modulated. ${ }^{[89,90]}$ Modulation of the firing frequency is possible via the excitation from parallel fibers and inhibition from inhibitory interneuron inputs. ${ }^{[91,92]}$ Indeed, this type of modulation of inhibitory neurons is not limited to mammals; it has recently been shown that the C. elegans AWA neuron is capable of firing an all-or-none calcium-based action potentials. ${ }^{[93]}$

Sensorimotor circuits are crucial to guide goal-oriented locomotion across evolution. Irrespective of the organism, the flow of information is preserved as the sensory input from the environment is processed by way of inhibitory interneuron gating (for detailed reviews, see Hennequin et al. ${ }^{[94]}$ and Sprekeler ${ }^{[95]}$. Before the motor output is generated, the information is further processed along with excitatory feedforward circuits whose activity is shaped by feed-forward and feed-back inhibitory projections. Such circuitry is likely to have appeared early during multicellular organisms' evolution and as organisms are exposed to new environments, the sensorimotor computation must also increase in complexity to allow existing networks to adapt and gain new functionality. ${ }^{[96]}$ The sensorimotor circuit organization across the evolutionary tree might serve as an example for the adaptive changes in circuit organization. Over time, the basic circuit elements evolve into more complex, parallel circuit loops that enable advanced multimodal computation, integration of mnemonic information, and executive control for top-down regulation of the 
sensorimotor control (see Figure 2 for a phylogenetic view on the circuits from the sea slug to fruit fly).

The sea slug, Hermissenda crassicornis (H. crassicornis), has one of the simplest invertebrate sensorimotor networks (Figure 2). Here, sensory information (light and vestibular) converges on the interneurons, but there is also reciprocal inhibition between the hair cells and photoreceptors. ${ }^{[1,4,6,97]}$ Such reciprocal inhibition is crucial for bilateral activity in invertebrates and vertebrates, including in C. elegans, where locomotion is accomplished by either inhibitory commissural neurons (CNs) acting directly on motor neurons (or interneurons) or indirectly via excitatory $\mathrm{CNs}$ acting on premotor inhibitory neurons. ${ }^{[110]}$ The axons of $\mathrm{CN}$ cross the midline and thus provide a direct line of communication, enabling changes in left-right coordination. ${ }^{[110]}$ Aquatic vertebrates, such as tadpoles and lampreys, also display the same bilateral action of CNs as observed in mammals. ${ }^{[110-113]}$

While interneurons allow computation of multimodal sensory integration in simple animals, such as the nematode, the presence of layers of network structures demonstrates more organization. Sensorimotor control loops are observed in the fly, where navigation is achieved via a combination of path integration (tracking position relative to a reference point) and known visual landmarks. ${ }^{[100]}$ Figure 2 shows a concise representation of the fly sensorimotor loops. ${ }^{[18,108,109]}$ The central complex (CX) is a point of convergence, having many inputs from sensorimotor circuits, with only a few outputs to guide flight pathways..$^{[100,101]}$ The CX is composed of a number of structures, including the protocerebral bridge $(\mathrm{PB})$, the fan-shaped body (FB), and the ellipsoid body as well as various substructures (Figure 2). Within this circuit, excitatory and inhibitory pathways create bidirectional circuits and contribute to sensorimotor control loops. ${ }^{[101]}$ Indeed, the PB is likely to be the location in which most sensorimotor computations occur and is analogous to the mammalian basal ganglia. ${ }^{[101]}$ Within the CX are a series of neurons arranged in a ring (ring neurons) that have both excitatory and inhibitory receptive fields (similar to cells in the mammalian primary sensory cortices). ${ }^{[100,102]}$

Ultimately, reciprocal inhibition is a computation common to all organisms throughout evolution, and inhibitory neurons remain central to the performance of such networks. As sensorimotor networks evolve, interneurons allow computation of multimodal sensory integration, as evidenced in the nematode, ${ }^{[68,77,114,115]}$ while in the fly, ${ }^{[79,80,100,101,116]}$ rodent, $^{[117]}$ and mammalian brain, ${ }^{[50-53,118-121]}$ the presence of layers of network structures demonstrates a hierarchical organization. Within this circuit, excitatory and inhibitory pathways create bidirectional circuits and contribute to sensorimotor control loops ${ }^{[83]}$ as convergent projections targeting the circuit enable the integration of multimodal bottom-up and top-down information while divergent projections enable modulation of a broad range of sensorimotor circuits throughout the brain.

Together, these observations suggest that independent of their position in the evolutionary tree, all animals are capable of using sensorimotor integration to generate context-specific actions, and this integration requires closed-loop computations. Inhibitory neurons are capable of shaping the control of movement and they function in parallel to, and as a part of, the
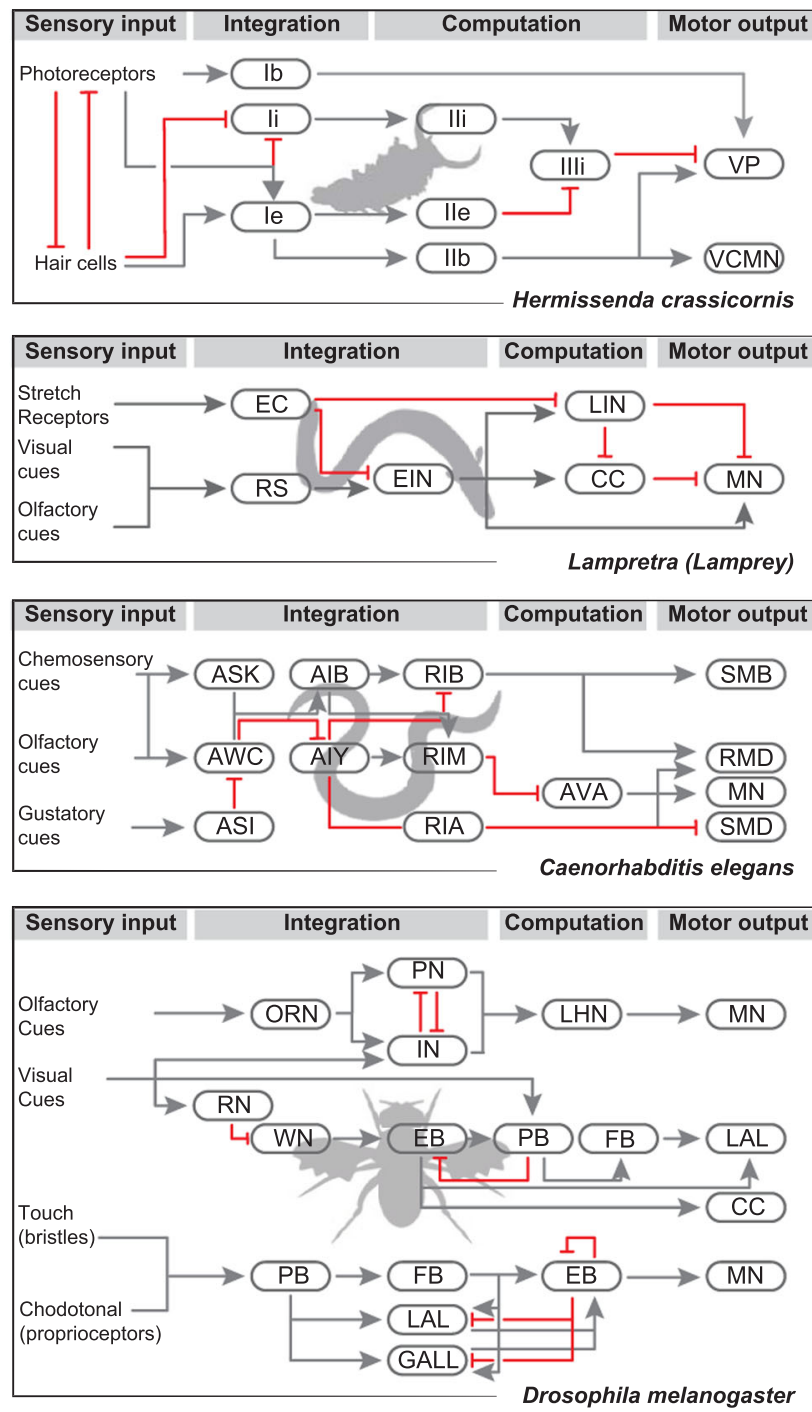

Figure 2. Sensorimotor computations require inhibition at all levels of neural circuits. Simplified circuit maps for four nonmammalian model organisms where sensorimotor transformations are experimentally well studied. The sea slug, $H$. crassicornis, has a simple nervous system. ${ }^{[1,6,97]}$ Inhibitory neurons are key in integrating information from the hair cells and photoreceptors to drive movement via cilia and tentacles. I, II, III, interneurons; VCMN, ventral contraction motor neuron; VP, ventral pedal. The lamprey requires inhibition to enable it to propagate forward movement via the contralateral activation and inhibition of body muscles. ${ }^{[98,99]} \mathrm{CC}$, crosscaudal inhibitor interneuron; $E D$, edge cell; EIN, excitatory interneuron; IIN, inhibitory interneuron; LIN, lateral interneuron; RS, reticulospinal cells. Similarly, C. elegans requires contralateral inhibition for the forward movement; see Figure 1 for more details. In contrast, Drosophila melanogaster is a much more complex system. ${ }^{[100-107]}$ Here, a combination of seeded sensorimotor loops and inhibition as well as compartmentalization enable the fly to integrate multiple senses in the $C X$ and respond accordingly. $F B$, fanshaped body; IN, interneuron; LAL, lateral accessory lobe; LHN, lateral horn neuron; MN, motor neuron; ORN, olfactory receptor neuron; $\mathrm{PB}$, protocerebral bridge; $\mathrm{PN}$, projection neuron; $\mathrm{RN}$, ring neuron; $\mathrm{WN}$, wedge neuron. Note that the sensorimotor circuit shown for the fly is concise and is not intended to represent the complete circuitry. ${ }^{[18,108,109]}$ 
seeded sensorimotor loops. Now, the challenge is to understand how the sensorimotor computation emerges from simple circuit elements as an emergent computation of the distributed networks in the brain. The ability to have independent control over each synaptic input to a cell/circuit component while monitoring the motor output will enable the computation of specific neuronal projections and provide causal links between neural activity and behavior.

\section{Conclusions and Future Perspectives}

Over the past century, scientists working on invertebrate animal models have unraveled the cellular atlas, especially in C. elegans, ${ }^{[122,123]}$ how cells interact across a network of brain regions, and which cell types encode the various sensory inputs. ${ }^{[124]}$ Now, we also have models of the mechanisms by which environmental cues are combined with goal-driven information to generate a spatial representation. ${ }^{[100-102]}$ However, questions remain concerning the contribution of higher-order processes, e.g., memory, to drive navigation in invertebrates. To answer these, we must leap from systems biology to systems neuroscience-with its quantitative framework-to causally link elementary neural circuit computations to behavior in freely behaving animals. The ultimate goal is to understand how complex behavior emerges from seemingly rudimentary neural processes and identify generalizable circuit computations that enable adaptive sensorimotor transformations in the context of navigation. The ability to have independent control over each synaptic input, including those of neuromodulatory transmitters, to a cell/circuit component while monitoring an output will address the computational roles of specific synaptic connections. This provides causal links between neural activity and behavior, and the availability of genetic tools will be crucial in working toward this goal. ${ }^{[78,93,124-128]}$

In understanding circuitry and by showing that the same processes apply to both simple model organisms (e.g., the fly and worm) as well as more complex mammalian species (mice and human), the scope for the inspection of how circuits function, signals are integrated, and loops function alone or in combination is significantly more achievable. Indeed, knowledge gleaned from this type of research will have profound implications in understanding the basis of sensorimotor deficits in neurological disorders and neurodegenerative disease. ${ }^{[129-131]}$ It might even provide a route to enable the development of bionic limbs as efficient sensorimotor control algorithms will facilitate rapid closed-loop control of artificial actuators.

\section{Acknowledgements}

The authors thank Dr. Erik Storkebaum and the members of the Department of Neurophysiology for discussions and their critical insight on a previous version of the manuscript.

\section{Conflict of Interest}

The authors declare no conflict of interest.

\section{Keywords}

Caenorhabditis elegans (C. elegans), inhibition, invertebrates, navigation, neural circuits

Received: May 29, 2019

Revised: July 17, 2019

Published online: August 21, 2019

[1] T. Crow, N. G. Jin, L.-M. Tian, J. Neurophysiol. 2013, 109, 640

[2] S. Leys, T. Cronin, B. Degnan, J. Marshall, J. Comp. Physiol., A 2002, 188, 199.

[3] G. Jékely, Proc. Biol. Sci. 2011, 278, 914.

[4] T. Crow, L.-M. Tian, J. Neurophysiol. 2008, 100, 2496.

[5] T. Crow, J. B. Redell, L.-M. Tian, J. Xue-Bian, P. K. Dash, J. Neurosci. 2003, 23, 3415.

[6] T. Crow, L.-M. Tian, J. Neurophysiol. 2003, 89, 2420.

[7] J. Voigts, D. H. Herman, T. Celikel, J. Neurophysiol. 2015, 113, 620.

[8] B. Zonooz, E. Arani, K. P. Körding, P. A. T. R. Aalbers, T. Celikel, A. J. van Opstal, Sci. Rep. 2019, 9, 1642.

[9] U. Górska, A. Rupp, Y. Boubenec, T. Celikel, B. Englitz, eNeuro 2018, 5, ENEURO.0090.

[10] J. Heckman, B. McGuinness, T. Celikel, B. Englitz, Neurosci. Biobehav. Rev. 2016, 65, 313.

[11] G. Konopka, T. F. Roberts, Cell 2016, 164, 1269.

[12] B. D. Kuebrich, S. J. Sober, Neuroscience 2015, 296, 48.

[13] K. Tschida, R. Mooney, Curr. Opin. Neurobiol. 2012, 22, 320.

[14] J. J. Heckman, R. Proville, G. J. Heckman, A. Azarfar, T. Celikel, B. Englitz, Sci. Rep. 2017, 7, 3017.

[15] T. Kitanishi, H. T. Ito, Y. Hayashi, Y. Shinohara, K. Mizuseki, T. Hikida, J. Physiol. Sci. 2017, 67, 247.

[16] A. W. Lester, S. D. Moffat, J. M. Wiener, C. A. Barnes, T. Wolbers, Neuron 2017, 95, 1019.

[17] S. J. Y. Mizumori, B. G. Cooper, S. Leutgeb, W. E. Pratt, Mol. Neurobiol. 2000, 21, 57.

[18] A. Honkanen, A. Adden, J. Da Silva Freitas, S. Heinze, J. Exp. Biol. 2019, 222, jeb188854.

[19] B. Webb, A. Wystrach, Curr. Opin. Insect Sci. 2016, 15, 27.

[20] C. Rochefort, J. Lefort, L. Rondi-Reig, Front. Neural Circuits 2013, 7, 35.

[21] P. A. Garrity, M. B. Goodman, A. D. Samuel, P. Sengupta, Genes Dev. 2010, 24, 2365.

[22] E. A. Kane, M. Gershow, B. Afonso, I. Larderet, M. Klein, A. R. Carter, B. L. de Bivort, S. G. Sprecher, A. D. T. Samuel, Proc. Natl. Acad. Sci. U. S. A. 2013, 110, E3868.

[23] C. I. Bargmann, in WormBook (Ed: T. C. e. R. Community), C. Elegans Research Community, Pasadena (CA) 2006.

[24] N. Vickers, Biol. Bull. 2000, 198, 203.

[25] W. Stein, J. Comp. Physiol., A 2009, 195, 989.

[26] C. F. Moss, C. Chiu, A. Surlykke, Curr. Opin. Neurobiol. 2011, 21, 645 .

[27] M. S. Smotherman, Behav. Brain Res. 2007, 182, 315.

[28] J. Voigts, B. Sakmann, T. Celikel, J. Neurophysiol. 2008, 100, 504.

[29] T. Celikel, B. Sakmann, Proc. Natl. Acad. Sci. U. S. A. 2007, 104, 1395.

[30] T. Celikel, V. Marx, F. Freudenberg, A. Zivkovic, E. Resnik, M. T. Hasan, P. Licznerski, P. Osten, A. Rozov, P. H. Seeburg, M. K. Schwarz, Front. Neurosci. 2007, 1, 97.

[31] N. S. Corsini, I. Sancho-Martinez, S. Laudenklos, D. Glagow, S. Kumar, E. Letellier, P. Koch, M. Teodorczyk, S. Kleber, S. Klussmann, B. Wiestler, O. Brüstle, W. Mueller, C. Gieffers, O. Hill, M. Thiemann, M. Seedorf, N. Gretz, R. Sprengel, T. Celikel, A. Martin-Villalba, Cell Stem Cell 2009, 5, 178.

[32] F. Freudenberg, E. Resnik, A. Kolleker, T. Celikel, R. Sprengel, P. H. Seeburg, Neurobiol. Learn. Mem. 2016, 135, 83. 
[33] F. Freudenberg, V. Marx, P. H. Seeburg, R. Sprengel, T. Celikel, Hippocampus 2013, 23, 1359.

[34] D. R. Albrecht, C. I. Bargmann, Nat. Methods 2011, 8, 599.

[35] P. Sengupta, A. D. Samuel, Curr. Opin. Neurobiol. 2009, 19, 637.

[36] H. C. Berg, D. A. Brown, Nature 1972, 239, 500.

[37] V. Sourjik, N. S. Wingreen, Curr. Opin. Cell Biol. 2012, 24, 262.

[38] E. Itskovits, R. Ruach, A. Zaslaver, Nat. Commun. 2018, 9, 2866.

[39] H. S. Kaplan, A. L. A. Nichols, M. Zimmer, Philos. Trans. R. Soc., B 2018, 373, 20170371

[40] A. Azarfar, Y. Zhang, A. Alishbayli, S. Miceli, L. Kepser, D. van der Wielen, M. van de Moosdijk, J. Homberg, D. Schubert, R. Proville, T. Celikel, Gigascience 2018, 7, giy 134.

[41] J. M. Gray, J. J. Hill, C. I. Bargmann, Proc. Natl. Acad. Sci. U. S. A. 2005, 102, 3184

[42] T. Wakabayashi, I. Kitagawa, R. Shingai, Neurosci. Res. Lett. 2004, 50,103

[43] J. Lim, T. Celikel, J. Neural. Eng. 2019.

[44] M. Zhen, A. D. Samuel, Curr. Opin. Neurobiol. 2015, 33, 117.

[45] J. A. Harris, C. Miniussi, I. M. Harris, M. E. Diamond, J. Neurosci. 2002, 22, 8720 .

[46] M. M. Hayhoe, Annu. Rev. Vision Sci. 2017, 3, 389.

[47] H. Makino, E. J. Hwang, N. G. Hedrick, T. Komiyama, Neuron 2016, 92, 705.

[48] R. Romo, L. Lemus, V. de Lafuente, Curr. Opin. Neurobiol. 2012, 22, 914.

[49] R. Rossi-Pool, J. Vergara, R. Romo, Trends Neurosci. 2018, 41, 117.

[50] D. J. Freedman, G. Ibos, Neuron 2018, 97, 1219.

[51] L. L. Richmond, J. M. Zacks, Trends Cognit. Sci. 2017, 21, 962.

[52] L. Rondi-Reig, A.-L. Paradis, J. M. Lefort, B. M. Babayan, C. Tobin, Front. Syst. Neurosci. 2014, 8, 205.

[53] K. Svoboda, N. Li, Curr. Opin. Neurobiol. 2018, 49, 33.

[54] K. M. Tabor, S. A. Bergeron, E. J. Horstick, D. C. Jordan, V. Aho, T. Porkka-Heiskanen, G. Haspel, H. A. Burgess, J. Neurophysiol. 2014, 112, 834

[55] M. A. Tanouye, R. J. Wyman, J. Neurophysiol. 1980, 44, 405.

[56] R. Dubuc, F. Brocard, M. Antri, K. Fénelon, J.-F. Gariépy, R. Smetana, A. Ménard, D. Le Ray, G. Viana Di Prisco, É. Pearlstein, M. G. Sirota, D. Derjean, M. St-Pierre, B. Zielinski, F. Auclair, D. Veilleux, Brain Res. Rev. 2008, 57, 172.

[57] D. D. Ghosh, T. Sanders, S. Hong, L. Y. McCurdy, D. L. Chase, N. Cohen, M. R. Koelle, M. N. Nitabach, Neuron 2016, 92, 1049.

[58] L. Wang, H. Sato, Y. Satoh, M. Tomioka, H. Kunitomo, Y. lino, J. Neurosci. 2017, 37, 2097.

[59] M. Hendricks, Y. Zhang, Worm 2013, 2, e25546.

[60] K. Kobayashi, S. Nakano, M. Amano, D. Tsuboi, T. Nishioka, S. Ikeda, G. Yokoyama, K. Kaibuchi, I. Mori, Cell Rep. 2016, 14, 11.

[61] A. D. T. Samuel, P. Sengupta, Curr. Biol. 2005, 15, R341.

[62] A. Kocabas, C.-H. Shen, Z. V. Guo, S. Ramanathan, Nature 2012, 490, 273.

[63] I. Mori, Y. Ohshima, Nature 1995, 376, 344.

[64] E. L. Tsalik, O. Hobert, Neurobiology 2003, 56, 178.

[65] O. Tolstenkov, P. van der Auwera, W. Steuer Costa, O. Bazhanova, T. M. Gemeinhardt, A. C. Bergs, A. Gottschalk, eLife 2019, 7, e34997.

[66] E. L. Ardiel, C. H. Rankin, Learn. Mem. 2010, 17, 191.

[67] H. Sasakura, I. Mori, Curr. Opin. Neurobiol. 2013, 23, 92.

[68] A. López-Cruz, A. Sordillo, N. Pokala, Q. Liu, P. T. McGrath, C. I. Bargmann, Neuron 2019, 102, 407.

[69] D. L. Glanzman, Curr. Biol. 2010, 20, pR31.

[70] M. Gomez, E. De Castro, E. Guarin, H. Sasakura, A. Kuhara, I. Mori, T. Bartfai, C. I. Bargmann, P. Nef, Neuron 2001, 30, 241.

[71] H.-S. Mun, B. J. Saab, E. Ng, A. McGirr, T. V. Lipina, Y. Gondo, J. Georgiou, J. C. Roder, Sci. Rep. 2015, 5, 17697.
[72] V. M. Martin, J. R. Johnson, L. P. Haynes, J. W. Barclay, R. D. Burgoyne, Mol. Brain 2013, 6, 39.

[73] Z. Li, J. Liu, M. Zheng, X. Z. S. Xu, Cell 2014, 159, 751.

[74] W. Zou, J. Fu, H. Zhang, K. Du, W. Huang, J. Yu, S. Li, Y. Fan, H. A. Baylis, S. Gao, R. Xiao, W. Ji, L. Kang, T. Xu, Nat. Commun. 2018, 9, 4311.

[75] M. Hendricks, H. Ha, N. Maffey, Y. Zhang, Nature 2012, 487, 99.

[76] A. Donato, K. Kagias, Y. Zhang, M. A. Hilliard, Biol. Rev. Cambridge Philos. Soc. 2019, 94, 1023.

[77] S. Kato, H. S. Kaplan, T. Schrödel, S. Skora, T. H. Lindsay, E. Yemini, S. Lockery, M. Zimmer, Cell 2015, 163, 656.

[78] S. W. Flavell, N. Pokala, E. Z. Macosko, D. R. Albrecht, J. Larsch, C. I. Bargmann, Cell 2013, 154, 1023.

[79] M. Joesch, B. Schnell, S. V. Raghu, D. F. Reiff, A. Borst, Nature 2010, 468, 300.

[80] M. S. Maisak, J. Haag, G. Ammer, E. Serbe, M. Meier A. Leonhardt, T. Schilling, A. Bahl, G. M. Rubin, A. Nern, B. J. Dickson, D. F. Reiff, E. Hopp, A. Borst, Nature 2013, 500, 212.

[81] T. Euler, P. B. Detwiler, W. Denk, Nature 2002, 418, 845.

[82] S. I. Fried, R. H. Masland, Curr. Biol. 2007, 17, R63.

[83] M. Lavzin, S. Rapoport, A. Polsky, L. Garion, J. Schiller, Nature 2012, 490, 397.

[84] J. Setschenow, in Physiologische studien uber den hemmungsmechanismus fur die reflexthatigkeit des ruckenmarkes und gehirnes der froschen, Hirschwald, Berlin 1863.

[85] E. G. T. Liddell, C. S. Sherrington, Proc. R. Soc. London, Ser. B 1925, 97, 488.

[86] C. S. Sherrington, Proc. R. Soc. London, Ser. B 1925, 97, 519.

[87] S. X. Chen, A. N. Kim, A. J. Peters, T. Komiyama, Nat. Neurosci. 2015, 18, 1109 .

[88] M. Goulding, S. Bourane, L. Garcia-Campmany, A. Dalet, S. Koch, Curr. Opin. Neurobiol. 2014, 26, 161.

[89] M. Ito, The Cerebellum and Neural Control, Raven Press, New York 1984

[90] J. S. Albus, Math. Biosci. 1971, 10, 25.

[91] A. Spanne, H. Jörntell, PLoS Comput. Biol. 2013, 9, e1002979.

[92] S. A. Heiney, J. Kim, G. J. Augustine, J. F. Medina, J. Neurosci. 2014, 34, 2321.

[93] Q. Liu, P. B. Kidd, M. Dobosiewicz, C. I. Bargmann, Cell 2018 175, 57.

[94] G. Hennequin, E. J. Agnes, T. P. Vogels, Annu. Rev. Neurosci. 2017, 40, 557.

[95] H. Sprekeler, Curr. Opin. Neurobiol. 2017, 43, 198.

[96] A. Azarfar, N. Calcini, C. Huang, F. Zeldenrust, T. Celikel, Neurosci. Biobehav. Rev. 2018, 94, 238

[97] T. Crow, L.-M. Tian, J. Neurophysiol. 2004, 91, 2874.

[98] A. El Manira, P. Wallén, News Physiol. Sci. 2000, 15, 186.

[99] J. T. Buchanan, Prog. Neurobiol. 2001, 63, 441.

[100] J. D. Seelig, V. Jayaraman, Nature 2013, 503, 262.

[101] C.-Y. Lin, C.-C. Chuang, T.-E. Hua, C.-C. Chen, B. J. Dickson, R. J. Greenspan, A.-S. Chiang, Cell Rep. 2013, 3, 1739.

[102] J. D. Seelig, V. Jayaraman, Nature 2015, 521, 186.

[103] A. Borst, Cold Spring Harbor Symp. Quant. Biol. 2014, 79, 131

[104] G. U. Busto, I. Cerbantes-Sandoval, R. L. Davis, Physiology 2010, $25,338$.

[105] N. J. Butcher, A. B. Friedrich, Z. Lu, H. Tanimoto, I. A. Meinertzhagen, J. Comput. Neurosci. 2012, 520, 2185.

[106] V. G. Fiore, R. J. Dolan, N. J. Strausfeld, F. Hirth, Philos. Trans. R. Soc., B 2015, 370, 20150053.

[107] A. Martín-Peña, A. Acebes, J.-R. Rodríguez, V. Chevalier, S. Casas-Tinto, T. Triphan, R. Strauss, A. Ferrús, Eur. J. Neurosci. 2014, 39, 1586.

[108] I. A. Meinertzhagen, J. Neurogenet. 2016, 30, 62 . 
[109] C.-T. Shih, O. Sporns, S.-L. Yuan, T.-S. Su, Y.-J. Lin, C.-C. Chuang, T.-Y. Wang, C.-C. Lo, R. J. Greenspan, A.-S. Chiang, Curr. Biol. 2015, 25, 1249.

[110] O. Kiehn, Nat. Rev. Neurosci. 2016, 17, 224.

[111] S. Grillner, Nat. Rev. Neurosci. 2003, 4, 573.

[112] S. Grillner, T. Deliagina, A. El Manira, R. H. Hill, G. N. Orlovsky, P. Wallén, Ö. Ekeberg, A. Lansner, Trends Neurosci. 1995, 18, 270.

[113] A. Roberts, W. C. Li, S. R. Soffe, J. Comp. Physiol., A 2008, 194, 185.

[114] D. D. Ghosh, M. N. Nitabach, Y. Zhang, G. Harris, Curr. Opin. Neurobiol. 2017, 43, 110.

[115] S. H. Chalasani, N. Chronis, M. Tsunozaki, J. M. Gray, D. Ramot, M. B. Goodman, C. I. Bargmann, Nature 2007, 450, 63.

[116] J. C. Tuthill, R. I. Wilson, Cell 2016, 164, 1046.

[117] N. J. Sofroniew, K. Svoboda, Curr. Biol. 2015, 25, R137.

[118] A. D. Redish, D. S. Touretzky, Hippocampus 1997, 7, 15.

[119] K. J. Jeffery, Hippocampus 2007, 17, 775.

[120] E. I. Moser, M.-B. Moser, B. L. McNaughton, Nat. Neurosci. 2017, $20,1448$.
[121] B. Poucet, E. Save, P.-P. Lenck-Santini, Rev. Neurosci. 2000, 11, 95.

[122] B. Mulcahy, D. Witvliet, D. Holmyard, J. Mitchell, A. D. Chisholm, Y. Meirovitch, A. D. T. Samuel, M. Zhen, Front. Neural Circuits 2018, 12, 94.

[123] S. J. Cook, T. A. Jarrell, C. A. Brittin, Y. Wang, A. E. Bloniarz, M. A. Yakovlev, K. C. Q. Nguyen, L. T. H. Tang, E. A. Bayer, J. S. Duerr, H. E. Bülow, O. Hobert, D. H. Hall, S. W. Emmons, Nature 2019, 571, 63.

[124] C. Barry, N. Burgess, Curr. Biol. 2014, 24, R330.

[125] D. A. Clark, L. Freifeld, T. R. Clandinin, Neuron 2013, 78, 583.

[126] K. J. T. Venken, J. H. Simpson, H. J. Bellen, Neuron 2011, 72, 202.

[127] C. Fang-Yen, M. J. Alkema, A. D. T. Samuel, Philos. Trans. R. Soc., B 2015, 370, 20140212.

[128] A. Gordus, N. Pokala, S. Levy, S. W. Flavell, C. I. Bargmann, Cell 2015, 161, 215.

[129] N. Patel, J. Jankovic, M. Hallett, Lancet Neurol. 2014, 13, 100.

[130] R. A. Grant, B. Mitchinson, C. W. Fox, T. J. Prescott, J. Neurophysiol. 2009, 101, 862.

[131] G. Abbruzzese, A. Berardelli, Mov. Disord. 2003, 18, 231. 